\title{
Optical conductivity in superconductors with mixed symmetry order parameters
}

\author{
I.Schürrer, E.Schachinger \\ Institut für Theoretische Physik, Technische Universität Graz, Petersgasse \\ 16, A-8010 Graz, Austria
}

Received April 21, 1998

\begin{abstract}
The real and imaginary part of the optical conductivity and the derived optical scattering rate of superconductors with mixed $s+d$ or $s+$ id symmetry order parameter is calculated for different mixing and for moderate impurity concentrations in two limiting cases, namely the Born (weak) and the resonant (unitary) scattering limit. There are pronounced differences in the optical conductivity between impurity limits and with the symmetry of the order parameter. Theoretical predictions are compared to experimental data.
\end{abstract}

Key words: high- $T_{\mathrm{c}}$ superconductivity, mixed symmetry, order-parameter, optical conductivity

PACS: 74.20.Fg, 74.25.Nf, 74.72.- $h$

\section{Introduction}

There is a number of experiments which indicate that the order parameter in some, if not in all, high temperature superconductors (HTCS) could be of mixed symmetry. Krishana et al. [1] investigated the electronic thermal conductivity of $\mathrm{Bi}_{2} \mathrm{Sr}_{2} \mathrm{CaCu}_{2} \mathrm{O}_{8}$ in a magnetic field profile experiment. They observed at non-zero magnetic fields a phase transition like behaviour and concluded that this could possibly be a phase transition from a simple $d$-wave to either a $d_{x^{2}-y^{2}}+\mathrm{i} d_{x y}$ or a $d_{x^{2}-y^{2}}+$ is symmetric order parameter. Kouznetsov et al. [2] offers direct evidence for a mixed order parameter in $\mathrm{YBa}_{2} \mathrm{Cu}_{3} \mathrm{O}_{6.95}$ (YBCO) using a new class of $c$-axis Josephson tunnelling experiments where the conventional superconductor $(\mathrm{Pb})$ is deposited across a single twin boundary. Their results are consistent with predominantly $d_{x^{2}-y^{2}}$ pairing symmetry and a sign reversal of a smaller $s$-wave component across the twin boundary.

In yet another experiment, using tunnelling spectroscopy, Covington et al. [3] observed surface-induced broken time-reversal symmetry in YBCO tunnelling junc- 
tions. This manifested itself by a split zero bias conductance peak. Such a result has been predicted theoretically by Fogelström et al. [4] as an Andreev bound state which is shifted to finite energy and this results in such a split zero bias conductance peak. This broken time-reversal symmetry state is identified as a $(d+\mathrm{i} s)$ symmetric state which develops in YBCO close to the boundary with an insulator.

Optical conductivity experiments seem to be becoming more and more important as a diagnostic tool in the field of high temperature superconductivity [5]. Palumbo and Graf [6] considered the case of pure $d_{x^{2}-y^{2}}$ symmetry, extended $s$-wave and anisotropic $s$-wave, and the effect of resonance impurity scattering on these. They find significant differences in the real part of the conductivity and discuss how to obtain information on the gap symmetry from data.

Here we will be interested in the optical conductivity of superconductors having an order parameter of either $(s+d)$ or $(s+\mathrm{i} d)$-symmetry as a function of increasing contributions of the $s$-wave symmetric part with the view of determining such an admixture from the experiment. We also consider and compare Born and resonant impurity scattering and concentrate on qualitative effects. Therefore, our calculations are restricted to the framework of the BCS-theory of superconductivity.

\section{Order parameter and crystal symmetry}

The dominant feature of HTCS are the $\mathrm{CuO}_{2}$ planes which are connected via atomic chains. These planes are commonly regarded to be most important for superconductivity. They are of tetragonal symmetry and for optimally doped systems the Fermi surface (FS) is a circle in the $\left(k_{x}, k_{y}\right)$-plane. (An example is, for instance, the material $\mathrm{Tl}_{2} \mathrm{Sr}_{2} \mathrm{CuO}_{6+\delta}$ (Tl2201).) The existence of $\mathrm{CuO}$ chains in YBCO means that it is orthorhombic and that large in-plane anisotropies are observed between the $x$ and $y$ (along the chains) axis in the optical conductivity [7].

The symmetry operations on the tetragonal lattice are as follows (besides the trivial identity operation): a) rotations by $90^{\circ}$ and $180^{\circ}$, and b) reflections along the $x$ and $y$-axis as well as along the diagonal of the unit cell. Thus we find five irreducible representations (table 1). An isotropic $s$-wave order parameter is the trivial choice because it is invariant under all operations and its FS average $\left\langle\Delta_{s}\right\rangle=$ const. Another possibility is the $d_{x^{2}-y^{2}}$ symmetry which requires the order parameter to change its sign along the FS which results in nodes on the FS and a $\left\langle\Delta_{d}\right\rangle=0$. The combination $(s+\mathrm{i} d)$-symmetry - there is a $90^{\circ}$ phase shift between the $s$ and the $d$-wave component of the order parameter - is also permitted. It has to be noted though that the $(s+\mathrm{i} d)$-symmetric order parameter breaks the time-reversal symmetry and cannot be expected to exist in the clean limit as far as pairing channels of different symmetry compete with each other and the dominant channel prevents the appearance of the subdominant one. On the other hand, due to pair breaking defects [8], like impurities in a $d$-wave superconductor, the dominant symmetry channel can be sufficiently suppressed to permit for a phase transition to either a mixed $(s+\mathrm{i} d)$-symmetric or even to a pure $s$-wave state. As we are not dealing with the clean limit in this paper it is quite realistic to study the possibility of an 
Table 1. Character table of the three dimensional space group of the tetragonal lattice.

\begin{tabular}{|c|rrrrr|l|l|}
\hline$C_{4 v}$ & $E$ & $2 C_{4}$ & $C_{2}$ & $2 \sigma_{v}$ & $2 \sigma_{d}$ & \multicolumn{2}{|l|}{} \\
\hline$A_{1}$ & 1 & 1 & 1 & 1 & 1 & $x^{2}+y^{2}, z^{2}$ & $s$-wave \\
$A_{2}$ & 1 & 1 & 1 & -1 & -1 & & \\
$B_{1}$ & 1 & -1 & 1 & 1 & -1 & $x^{2}-y^{2}, z^{2}$ & $d_{x^{2}-y^{2}-\text { wave }}$ \\
$B_{2}$ & 1 & -1 & 1 & -1 & 1 & $x y$ & $d_{x y}$-wave \\
$E$ & 2 & 0 & -2 & 0 & 0 & & \\
\hline
\end{tabular}

$(s+\mathrm{i} d)$-symmetric order parameter in a superconductor of tetragonal symmetry and how it affects the optical conductivity.

The situation changes drastically for orthorhombic systems with their $(x, y)$-anisotropy. The rotation by $90^{\circ}$ and the reflection along the diagonal are no longer permitted as symmetry operations. Therefore, it becomes impossible to distinguish between the isotropic $s$ and a $d_{x^{2}-y^{2}}$-symmetric order parameter from a group theoretical point of view. Thus, both are in the same irreducible representation and can of course mix freely leading to an $(s+d)$ symmetry. Moreover, the simplest approach to band anisotropy in orthorhombic systems is a free electron infinite band model but with different effective masses along the $x$ - and $y$-directions. This results in an ellipsoidal FS as opposed to the circular FS in systems of tetragonal symmetry.

\section{Formalism}

\subsection{Tetragonal symmetry}

We define the anisotropic, mixed symmetry order parameter $\Delta_{s+\mathrm{id}}(\varphi)$ in the clean limit by

$$
\Delta_{s+\mathrm{i} d}(\varphi)=\Delta_{s}+\mathrm{i} \Delta_{d}(\varphi)
$$

with $\Delta_{s}$ the $s$-wave contribution and $\Delta_{d}(\varphi)=\Delta_{d} \sqrt{2} \cos 2 \varphi$ the $d$-wave component of $d_{x^{2}-y^{2}}$-symmetry. Furthermore, we concentrate on the low temperature regime which makes it unnecessary to solve the BCS gap equation explicitly. Instead, we choose the clean limit, zero temperature order parameter $\Delta_{0}$ by either using the BCS relation $2 \Delta_{0} / k_{\mathrm{B}} T_{\mathrm{c}}=3.57$ (or $2 \sqrt{2} \Delta_{0} / k_{\mathrm{B}} T_{\mathrm{c}}=4.29$ for pure $d$-wave) or by choosing an appropriate experimental value.

The $\Delta_{s}$ to $\Delta_{d}$ admixture in equation (1) can be defined by introducing a parameter $\gamma$ in such a way that

$$
\Delta_{s}=\gamma \Delta_{0}, \quad \Delta_{d}=\sqrt{1-\gamma^{2}} \Delta_{0}
$$

which ensures that

$$
\Delta_{0}=\sqrt{\left\langle\Delta_{s+\mathrm{i} d}^{2}(\varphi)\right\rangle_{\varphi}}
$$

with $\langle\cdots\rangle_{\varphi}$ the FS-average. Thus, $0 \leqslant \gamma \leqslant 1$ with $\gamma=0$ the pure $d$-wave and $\gamma=1$ 
the pure $s$-wave case. An even more convenient parameter is

$$
x=\frac{\gamma}{\gamma+\sqrt{1-\gamma^{2}}}
$$

which gives, when multiplied by 100 , the percentage of $s$-wave gap $\Delta_{s}$, contained in $\Delta_{s+\mathrm{i} d}(\varphi)$.

Following the standard BCS theory, the quasiparticle propagator $G(\mathbf{k}, \nu)$ is determined by Dyson's equation

$$
G^{-1}(\mathbf{k}, \nu)=\nu \tau_{0}+\varepsilon_{\mathbf{k}} \tau_{3}-\Sigma_{\mathrm{BCS}}-\Sigma_{\mathrm{imp}}
$$

in a Nambu notation. Here, $\tau_{0}$ is the unity matrix, $\tau_{i}, i=1,2,3$ are the Pauli matrices which take care of the particle hole symmetry, $\varepsilon_{\mathbf{k}}$ is the quasiparticle dispersion relation, $\Sigma_{\mathrm{BCS}}$ is the BCS self energy contribution describing the correlation of quasiparticles in Cooper pairs. Included is also $\Sigma_{\text {imp }}$, the self energy which accounts for the quasiparticle impurity scattering. According to Ambegaokar [9] $\Sigma_{\text {BCS }}$ and $\Sigma_{\text {imp }}$ can be combined into one renormalized BCS self energy contribution

$$
\tilde{\Sigma}_{\mathrm{BCS}}=\nu-\tilde{\omega}-\left(\Delta_{1}-\tilde{\Delta}_{1}\right) \tau_{1}+\left(\Delta_{2}-\tilde{\Delta}_{2}\right) \tau_{2}
$$

with $\Delta_{1}$ and $\Delta_{2}$ the real and imaginary part of the clean limit order parameter, and $\tilde{\omega}, \tilde{\Delta}_{1}$, and $\tilde{\Delta}_{2}$ yet unknown complex functions. If we identify $\Delta_{1}$ with $\Delta_{s}$ and $\Delta_{2}$ with $\Delta_{d}(\varphi)$ we find the following renormalization equations in the weak (Born) impurity scattering approximation for dilute impurity concentrations $n_{\mathrm{I}}$ [10]:

$$
\begin{aligned}
\tilde{\omega}(\nu) & =\nu+\mathrm{i} \pi t^{+}\left\langle\frac{\tilde{\omega}(\nu)}{\sqrt{\tilde{\omega}^{2}(\nu)-\tilde{\Delta}_{s}^{2}(\nu)-\tilde{\Delta}_{d}^{2}(\varphi)}}\right\rangle \\
\tilde{\Delta}_{s}(\nu) & =\Delta_{s}+\mathrm{i} \pi t^{+}\left\langle\frac{\tilde{\Delta}_{s}(\nu, \varphi)}{\sqrt{\tilde{\omega}^{2}(\nu)-\tilde{\Delta}_{s}^{2}(\nu)-\tilde{\Delta}_{d}^{2}(\varphi)}}\right\rangle_{\varphi}, \quad \tilde{\Delta}_{d}(\varphi)=\Delta_{d}(\varphi),
\end{aligned}
$$

with $\tilde{\Delta}_{d}(\varphi)=\tilde{\Delta}_{d} \sqrt{2} \cos 2 \varphi \cdot t^{+}=n_{\mathrm{I}} N(0)\left|V\left(k_{\mathrm{F}}\right)\right|^{2}, \mathrm{~N}(0)$ is the normal state quasiparticle density of states at the Fermi energy, and $V\left(k_{\mathrm{F}}\right)$ is the scattering potential evaluated at the Fermi momentum $k_{\mathrm{F}}$. By the square root is meant the branch of the complex function which corresponds to a positive imaginary part.

In the more general case of arbitrary impurity scattering, strength $t^{+}$is to be replaced by $\Gamma^{+}=n_{\mathrm{I}} / N(0) \pi^{2}$ and the renormalization equations are modified:

$$
\begin{aligned}
& \tilde{\omega}(\nu)=\nu+\mathrm{i} \pi \Gamma^{+} \frac{\Omega(\nu)}{c^{2}+\Omega^{2}(\nu)+D^{2}(\nu)}, \\
& \tilde{\Delta}_{s}(\nu)=\Delta_{s}+\mathrm{i} \pi \Gamma^{+} \frac{D(\nu)}{c^{2}+\Omega^{2}(\nu)+D^{2}(\nu)}, \quad \tilde{\Delta}_{d}(\varphi)=\Delta_{d}(\varphi),
\end{aligned}
$$

with $c=\cot \delta_{0}$ and $\delta_{0}$ the T-matrix phase shift. For very large values of $c$ the results (7) and (8) for Born scattering are recovered and for $c=0$ we are in the so-called 
unitary (resonant) scattering limit. We also introduced the abbreviations

$$
\begin{aligned}
& \Omega(\nu)=\left\langle\frac{\tilde{\omega}(\nu)}{\sqrt{\tilde{\omega}^{2}(\nu)-\tilde{\Delta}_{s}^{2}(\nu)-\tilde{\Delta}_{d}^{2}(\varphi)}}\right\rangle_{\varphi}, \\
& D(\nu)=\left\langle\frac{\tilde{\Delta}_{s}(\nu)}{\sqrt{\tilde{\omega}^{2}(\nu)-\tilde{\Delta}_{s}^{2}(\nu)-\tilde{\Delta}_{d}^{2}(\varphi)}}\right\rangle_{\varphi} .
\end{aligned}
$$

\subsection{Orthorhombic symmetry}

The order parameter of a two dimensional Fermi liquid which is of $(s+d)$ symmetry can be described by [11]:

$$
\Delta(\varphi)=\Delta_{0}(\cos 2 \varphi+\beta),
$$

where $\beta$ describes the $s$-wave contribution to the dominant $d$-wave part which is modeled according to $d_{x^{2}-y^{2}}$ symmetry; $\varphi$ is the polar angle in the two dimensional Brillouin zone. The Fermi energy $\varepsilon_{\mathrm{F}}$ of the free electron infinite band model is given by

$$
\varepsilon_{\mathrm{F}}=\frac{k_{\mathrm{F}, x}^{2}}{2 m_{x}}+\frac{k_{\mathrm{F}, y}^{2}}{2 m_{y}},
$$

with $k_{\mathrm{F},(x, y)}$ the Fermi momenta in $x$ and $y$-direction respectively, and $m_{(x, y)}$ the corresponding effective masses.

This ellipsoidal Fermi surface can be transformed into a circular one through the transformation from $\left(k_{i}, \varphi\right)$ to $\left(p_{i}, \phi\right)$ space

$$
\begin{aligned}
p_{i} & =k_{i} \sqrt{\frac{m_{x}+m_{y}}{2 m_{i}}}, \quad i=x, y, \\
\tan \phi & =\sqrt{\frac{m_{y}}{m_{x}}} \tan \varphi .
\end{aligned}
$$

We introduce the mass anisotropy parameter

$$
\alpha=\frac{m_{x}-m_{y}}{m_{x}+m_{y}}
$$

with $\alpha \geqslant 0$, needed to describe YBCO because the chain direction has the smaller effective mass and is along $y$. In this new system of coordinates the gap (13) transforms into

$$
\begin{aligned}
\Delta(\phi) & =\Delta_{0}(f(\phi)+\beta) \\
& =\Delta_{0}\left(\frac{\cos 2 \phi+\alpha}{1+\alpha \cos 2 \phi}+\beta\right),
\end{aligned}
$$

which is no longer of $d$-wave symmetry. Nevertheless, it is possible to transform (18) into a form which corresponds to an $(s+d)$-model with one component $\Delta_{d}(\phi)$ which 
contains all the angular dependence and which also has the property $\left\langle\Delta_{d}(\phi)\right\rangle_{\phi}=0$ and a second component $\Delta_{s}$ which has no angular dependence. We introduce

$$
\bar{f}=\frac{1}{2 \pi} \int_{0}^{2 \pi} \mathrm{d} \phi f(\phi)=\frac{1-\sqrt{1-\alpha^{2}}}{\alpha} .
$$

This results in the two components of the order parameter:

$$
\begin{aligned}
\Delta_{d}(\phi) & =\frac{\Delta_{0}}{\sqrt{N}}(f(\phi)-\bar{f}), \\
\Delta_{s} & =\frac{\Delta_{0}}{\sqrt{N}}(\bar{f}+\beta), \\
N & =\left\langle(f(\phi)+\beta)^{2}\right\rangle_{\phi} .
\end{aligned}
$$

The normalization factor $N$ ensures that $\Delta_{0}=\sqrt{\left\langle\Delta_{s+d}^{2}(\phi)\right\rangle_{\phi}}$. In this notation equations (9) and (10) are again valid but now with

$$
\begin{aligned}
& \Omega(\nu)=\left\langle\frac{\tilde{\omega}(\nu)}{\sqrt{\tilde{\omega}^{2}(\nu)-\tilde{\Delta}_{s+d}^{2}(\nu, \phi)}}\right\rangle_{\phi}, \\
& D(\nu)=\left\langle\frac{\tilde{\Delta}_{s+d}(\nu, \phi)}{\sqrt{\tilde{\omega}^{2}(\nu)-\tilde{\Delta}_{s+d}^{2}(\nu, \phi)}}\right\rangle_{\phi} .
\end{aligned}
$$

and $\tilde{\Delta}_{s+d}(\nu, \phi)=\tilde{\Delta}_{s}(\nu)+\tilde{\Delta}_{d}(\nu, \phi)$. The addition of an $s$-wave component $(\beta \neq 0)$ to the gap or the inclusion of mass anisotropy $\left(m_{x} \neq m_{y}, \alpha \neq 0\right)$ can be referred to as orthorhombic $d$-wave symmetry.

\subsection{Optical conductivity}

The optical conductivity describes the linear response of a material which is exposed to an electromagnetic field. This field induces shielding currents

$$
J_{\mu}(\mathbf{q}, \nu)=-\sum_{\rho} K_{\mu \rho}(\mathbf{q}, \nu) A_{\rho}(\mathbf{q}, \nu), \quad \rho, \mu=0,1,2,3
$$

with $\nu$ the photon energy, $A_{\rho}(\mathbf{q}, \nu)$ the Fourier transform of the covariant vector potential and $K_{\mu \rho}(\mathbf{q}, \nu)$ the response kernel which depends only on the properties of the material. It can be expressed as a product of quasiparticle propagators $G(\mathbf{k}, \nu)$ and once this is known, the optical conductivity follows from

$$
\sigma(\nu)=\frac{\mathrm{i}}{\nu} K(\nu)
$$

after a q-integration. The quasiparticle propagator is calculated from equation (5) using either equations $(9-12)$ for the $(s+\mathrm{id})$ symmetry or equations $(9,10,23,24)$ for 
the orthorhombic $d$-wave case. The response kernel in (25) has first been calculated by Lee et al. [12] for superconductors with arbitrary impurity concentrations and it has been modified by Schürrer et al. $[10,11]$ to encompass mixed symmetry order parameters.

We find the isotropic conductivity in a tetragonal system by

$$
\sigma(\nu)=\frac{\mathrm{i}}{\nu} \frac{e^{2} N(0) v_{\mathrm{F}}^{2}}{2}\langle S(\nu, \varphi)\rangle_{\varphi}
$$

with $e$ the charge on the electron, $v_{\mathrm{F}}$ the Fermi velocity, and $S(\nu, \varphi)$ according to [10] equation (19). For the orthorhombic system Schürrer et al. [11] quote

$$
\begin{aligned}
\sigma_{x x^{\prime}}(\nu) & =\frac{\mathrm{i}}{\nu} e^{2} N_{n}(0) \frac{v_{\mathrm{F}}^{2}}{1+\alpha}\left\langle\cos ^{2} \phi S(\nu, \phi)\right\rangle_{\phi}, \\
\sigma_{y y^{\prime}}(\nu) & =\frac{\mathrm{i}}{\nu} e^{2} N_{n}(0) \frac{v_{\mathrm{F}}^{2}}{1-\alpha}\left\langle\sin ^{2} \phi S(\nu, \phi)\right\rangle_{\phi},
\end{aligned}
$$

for the anisotropic optical conductivity.

\section{Results}

Optical conductivity experiments study the excitation of quasiparticles from energies below the Fermi level into empty states above this level by photons of energy $\nu$. Therefore, the quasiparticle density of states (QDOS) $N(\nu)$ is reflected in $\sigma(\nu)$ and this makes the optical conductivity such a successful probe of the QDOS of complex quasiparticle systems.

We present in this chapter the results of numerical calculations for superconductors with Born impurity scattering (for instance dilute concentrations of impurities caused by radiation damage) and for superconductors which contain resonant scatterers (for instance $\mathrm{Zn}$ atoms substituted on $\mathrm{Cu}$-sites). The impurity concentration is either determined by the parameter $t^{+} / \Delta_{0}=0.0083$ (Born limit) or by $\Gamma^{+} / \Delta_{0}=0.0083$ (unitary limit). This ensures the same $T_{\mathrm{c}}$ in all systems. All calculations have been performed for a sample temperature of $10 \mathrm{~K}$.

\section{1. $(s+\mathrm{i} d)$ symmetry}

The quasiparticle density of states $N(\nu)$ normalized to its normal state value at the Fermi level $N(0)$ is calculated from

$$
\frac{N(\nu)}{N(0)} \propto\left\langle\Re \mathrm{e}\left(\frac{\tilde{\omega}\left(\nu+\mathrm{i} 0^{+}\right)}{\sqrt{\tilde{\omega}^{2}\left(\nu+\mathrm{i} 0^{+}\right)-\tilde{\Delta}_{s}^{2}\left(\nu+\mathrm{i} 0^{+}\right)-\tilde{\Delta}_{d}^{2}\left(\nu+\mathrm{i} 0^{+}, \varphi\right)}}\right)\right\rangle,
$$

with $\tilde{\omega}\left(\nu+\mathrm{i} 0^{+}\right), \tilde{\Delta}_{s}\left(\nu+\mathrm{i} 0^{+}\right)$, and $\tilde{\Delta}_{d}\left(\nu+\mathrm{i} 0^{+}, \varphi\right)$ determined from equations (9-12) for Born impurity scattering (top frame) or by equations (23) and (24) for resonant scatterers (bottom frame) respectively. It is obvious from equation (30) that the 

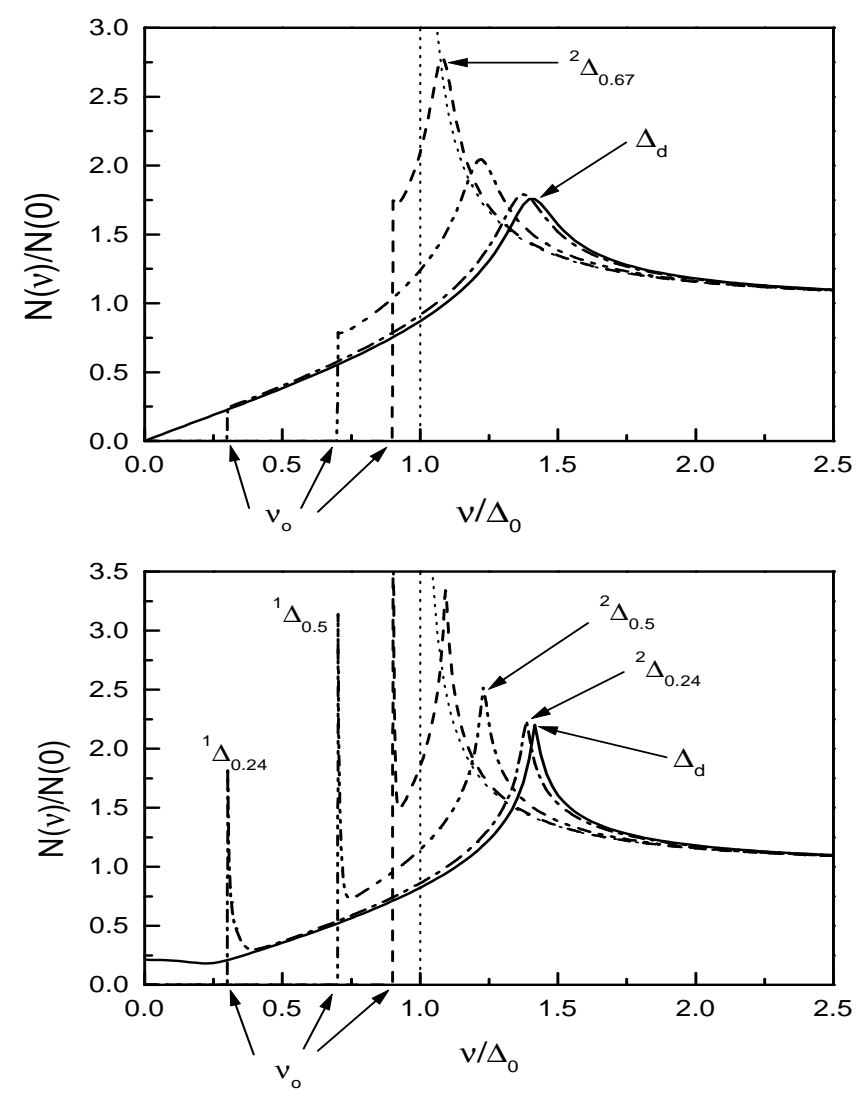

Figure 1. The superconducting QDOS $N(\nu)$ normalized to its normal state value at the Fermi level $N(0)$, as a function of the reduced energy $\nu / \Delta_{0}$ for a system with an $(s+\mathrm{i} d)$-symmetric order parameter, for various values of $x$. The top frame is for a system with Born impurity scattering and the bottom frame is for a system with resonant impurity scattering. The various $x$ values are: $x=0$ (pure $d$-wave, solid line), $x=0.24$ (dash-dotted line), $x=0.5$ (dash-double-dotted line), $x=0.67$ (dashed line), and $x=1$ (thin, dotted line).

QDOS has to have a gap for all $x>0$ because the real and imaginary parts of $\tilde{\Delta}_{s}^{2}$ and $\tilde{\Delta}_{d}^{2}$ never vanish simultaneously. Results are presented in figure 1 where the QDOS $N(\nu)$ normalized to its normal state value $N(0)$ is presented as a function of the reduced photon energy $\nu / \Delta_{0}$. Both frames contain as a reference the QDOS of a pure $s$-wave (dotted) and of a pure $d$-wave (solid) superconductor. The top frame of figure 1 presents the results of a superconductor which contains Born impurity scattering. We observe one peak in the QDOS which can be found at $1<\nu / \Delta_{0}<\sqrt{2}$ for $0<x<1$ and there is always a gap in the QDOS in the region $0 \leqslant \nu / \Delta_{0} \leqslant \alpha$ with a sharp rise in the QDOS at $\nu=\nu_{o}$, the onset energy.

An interesting situation arises when the superconductor contains resonant impurity scattering. These impurities create localized states which cause a finite QDOS at $\nu=0$ in the pure $d$-wave superconductor (solid line, bottom frame of figure 1). In 

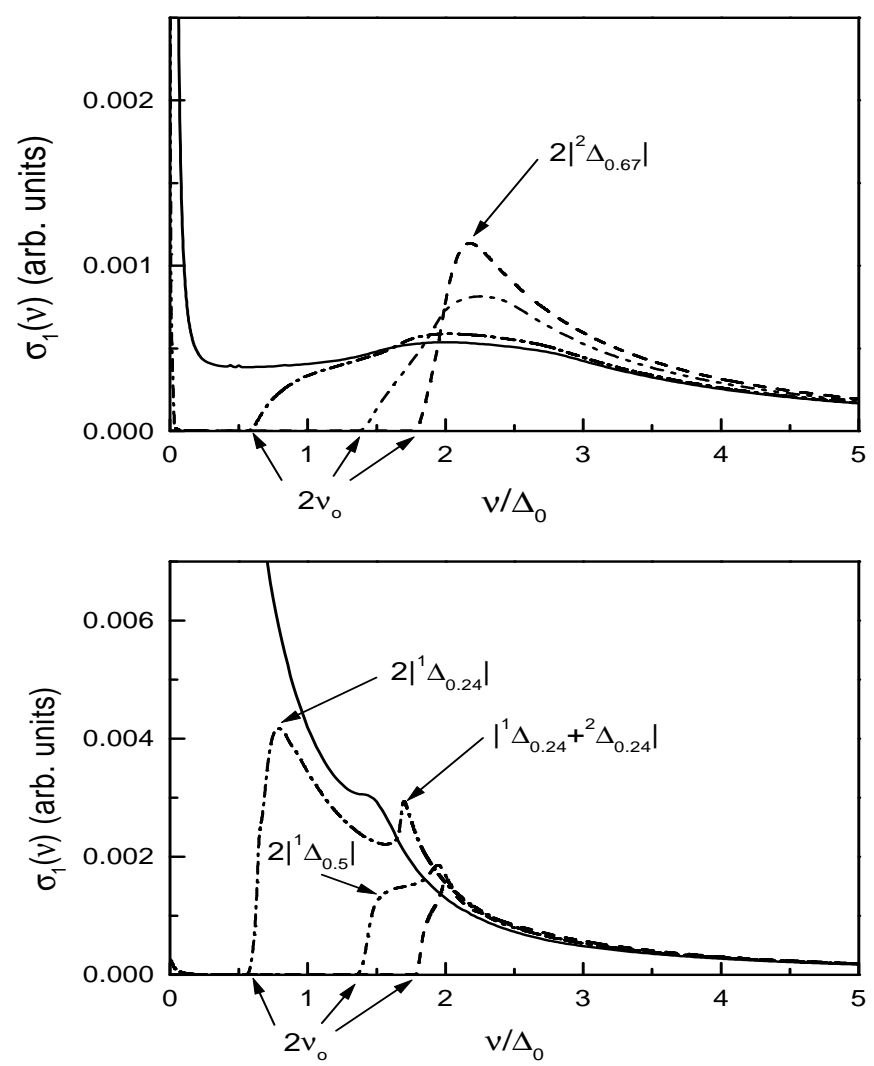

Figure 2. The real part of the optical conductivity $\sigma_{1}(\nu)$ in arbitrary units as a function of the reduced energy $\nu / \Delta_{0}$ for a system with $(s+\mathrm{i} d)$-symmetric order parameter. Various values of the parameter $x$ are considered, namely $x=0$ (pure $d$-wave, solid line), $x=0.24$ (dash-dotted line), $x=0.5$ (dash-double-dotted line), and $x=0.67$ (dashed line). The top frame is for Born impurity scattering and the bottom frame is for resonant scatterers.

the system with an $(s+\mathrm{i} d)$-symmetric order parameter such localized states cannot be created at $\nu=0$ because of the $(s+\mathrm{i} d)$ symmetry requirements and, therefore, they "pile up" at the onset frequency $\nu_{o}$ creating a sharp peak in the QDOS quite similar to the well known square root singularity at the gap of a standard $s$ wave superconductor. A second peak in the QDOS is again found at the frequencies $1<\nu / \Delta_{0}<\sqrt{2}$ for $0<x<1$.

The real part of the optical conductivity $\sigma_{1}(\nu)$ is presented in figure 2 as a function of the reduced frequency $\nu / \Delta_{0}$. As a reference both frames contain the results for a $d$-wave superconductor (solid lines). In the case of Born impurity scattering the QDOS has no gap and, therefore, even photons of infinitesimal energy will be capable of exciting some quasiparticles from the occupied to the unoccupied parts of the QDOS. We observe a very narrow peak structure of finite width around $\nu=0$. In addition there is a $\delta$-function peak at $\nu=0$ which corresponds to the condensate 
and gives the infinite d.c. conductivity of the superconductor. This is not shown here. The absorptive part of the conductivity stays finite for all energies above the Drude peak centered at $\nu=0$ and shows a second maximum at $\nu=2\left|\Delta_{d}\right|=2 \sqrt{2} \Delta_{0}$ (although it is not very pronounced). For even higher energies, $\sigma_{1}(\nu)$ approaches its normal state value.

As far as the QDOS for $x>0$ always has got a gap, the peak in $\sigma_{1}(\nu)$ around $\nu=0$ is very small at $T=10 \mathrm{~K}$ because few quasiparticles are thermally activated at such temperatures and so this peak can hardly be seen in the figure which shows a region of nearly zero conductivity, i.e.: $\sigma_{1}(\nu)$ exhibits a gap for frequencies smaller than two times the QDOS onset frequency $\nu_{o}$. In superconductors which contain Born impurity scattering, $\sigma_{1}(\nu)$ increases with increasing frequency for $\nu>\nu_{o}$ until a peak is reached near or at twice the frequency at which the QDOS has got its main peak. (For instance at $\nu=\left.2\right|^{2} \Delta_{0.67} \mid$ for $x=0.67$, dashed line in the top frame of figure 2.)

Superconductors which contain resonant impurity scattering behave quite differently as is demonstrated by our results presented in the bottom frame of figure 2 . For the pure $d$-wave case there is now a finite QDOS at $\nu=0$ (see bottom frame of figure 1, solid line). These electrons are easily excited and can absorb a photon directly just as in a normal metal. The result is a broad Drude like peak (solid line in the bottom frame of figure 2) centered around $\nu=0$. Direct absorption from the $\nu \approx 0$ region to the peak in the QDOS will now lead to the structure at the peak frequency rather than twice this value as has been seen in the Born impurity scattering case. For $x>0 \sigma_{1}(\nu)$ develops a steep rise at $\nu=\nu_{o}$ followed by either a pronounced peak or a shoulder indicating the availability of large numbers of free states because of the peak structure in the QDOS at the onset frequency $\nu_{o}$. See for instance the peak at $\nu=\left.2\right|^{1} \Delta_{0.24} \mid(x=0.24$, dash-dotted line $)$ or the shoulder at $\nu=\left.2\right|^{1} \Delta_{0.5} \mid$ $(x=0.5$, dash-double-dotted line). This first peak (shoulder) is then followed by a second, less pronounced peak which corresponds to excitation processes from states at the onset frequency below the Fermi level into free states at the main peak of the QDOS above the Fermi level. For $x=0.24$, this peak is found at the frequency $\nu=\left|{ }^{1} \Delta_{0.24}+{ }^{2} \Delta_{0.24}\right|$.

A comparison of experimental data for an optimally doped Tl2201 single crystal with theoretical predictions for a superconductor of $(s+\mathrm{i} d)$-symmetry is presented in figure 3. What is shown is the optical scattering rate of the superconducting state

$$
\frac{1}{\tau_{S}(\nu)}=\frac{\omega_{p}}{4 \pi} \Re \mathrm{e}\left[\frac{1}{\sigma(\nu)}\right]
$$

normalized to its frequency independent normal state value $\tau_{N}^{-1}$ as a function of the reduced energy $\nu / \Delta_{0}$. ( $\omega_{p}$ is the plasma frequency.) Experimental data are indicated by solid squares. They were read off figure 9 of [14] and rescaled in energy to match the main rise in the theoretical data. These are presented for $x=0$ (pure $d$-wave, solid line), $x=0.24$ (dash-dotted line), and $x=0.5$ (dashed line). We conclude from this comparison that the order parameter of Tl2201 is most likely of pure $d$-wave symmetry because no gap opens up in the $\tau_{S}^{-1}$ data as it would be required if there were a finite $s$-wave contribution to the order parameter. 


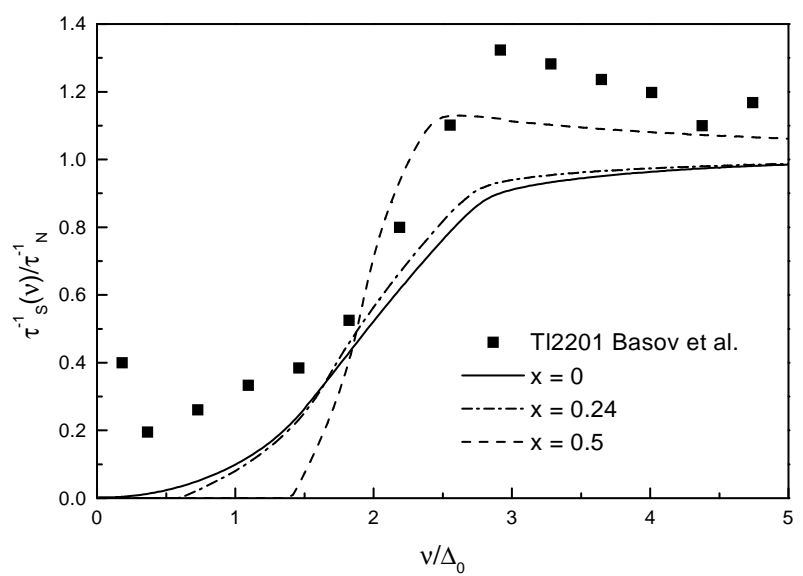

Figure 3. The superconducting state optical scattering rate $\tau_{S}^{-1}(\nu)$ normalized to its normal state value $\tau_{N}^{-1}$ as a function of the reduced energy $\nu / \Delta_{0}$. Theoretical predictions for an $(s+\mathrm{i} d)$-symmetric order parameter are compared to experimental data for a nominally clean, optimally doped Tl2201 single crystal (solid squares) [13]. The various $x$ values are: $x=0$ (pure $d$-wave, solid line), $x=0.24$ (dashed line), and $x=0.5$ (dash-dotted line). (The data points have been read off figure 9 of [13].) The energy scale of the experimental data was rescaled to match the main rise with theoretical predictions.

\subsection{Orthorhombic $d$-wave}

The function $f(\phi)$, equation (18), is central to understanding the effect of effective mass anisotropy on the resulting superconducting properties. The maxima and minima of $f(\phi)$ fall at 0 and $\pi$ and $\pi / 2$ and $3 \pi / 2$ respectively as they would in the pure $d$-wave case. The absolute magnitude of $f(\phi)$ is the same at both extrema. Therefore, the introduction of an effective mass anisotropy will not result in a splitting of the van Hove singularity in the QDOS positioned in energy at the value of the gap extrema. Only for $\beta \neq 0$ a splitting of the van Hove singularity can be expected.

In figure 4 (top and bottom frame) we consider the QDOS defined by

$$
\frac{N(\nu)}{N(0)} \propto\left\langle\Re \mathrm{e}\left(\frac{\tilde{\omega}\left(\nu+\mathrm{i} 0^{+}\right)}{\sqrt{\tilde{\omega}^{2}\left(\nu+\mathrm{i} 0^{+}\right)-\tilde{\Delta}_{s+d}^{2}\left(\nu+\mathrm{i} 0^{+}, \phi\right)}}\right)\right\rangle_{\phi},
$$

as a function of the reduced energy $\nu / \Delta_{0}$ for four different values of $\alpha$ in the top frame, and for $\alpha=0.4$ and three different values of $\beta$ in the bottom frame. The impurity scattering is in the resonant scattering limit. In both frames dotted, solid, dashed and dash-dotted curves are presented. In the top frame only effective mass anisotropy is included and $\alpha=0,0.2,0.4$, and 0.6 respectively. In the bottom frame the $s$-wave admixture represented by the parameter $\beta$ of equation (13) is $\beta=0.4$, 

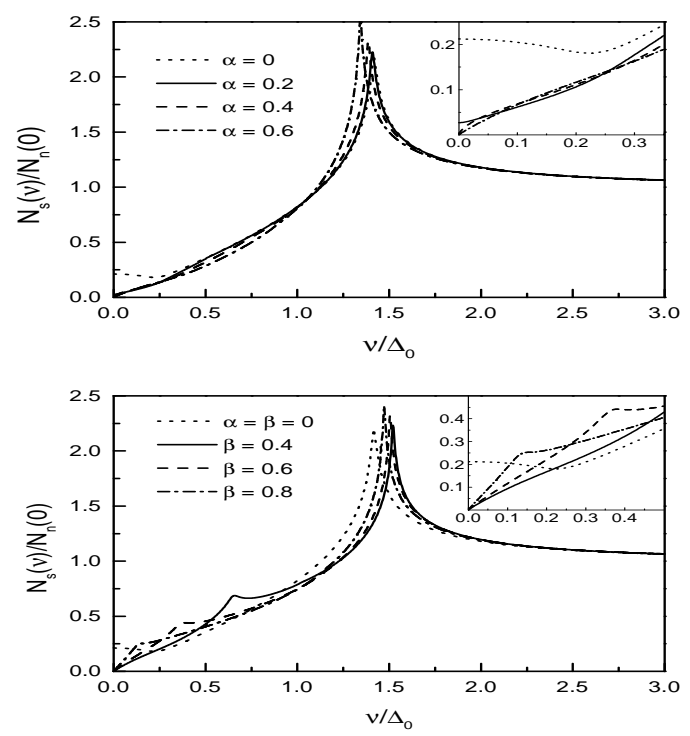

Figure 4. The superconducting state QDOS $N(\nu)$ normalized to its normal state value at the Fermi energy $N(0)$ as a function of the reduced energy $\nu / \Delta_{0}$ in a system with resonant impurity scattering. The top frame is for a pure $d$-wave gap but with orthorhombic band structure. The dotted curve is for $\alpha=0$, no band anisotropy, solid is for $\alpha=0.2$, dashed is for $\alpha=0.4$, and dash-dotted is for $\alpha=0.6$. Band structure anisotropy, i.e. $\alpha \neq 0$, rapidly degrades the finite value of $N(\nu) / N(0)$ in the $\nu \rightarrow 0$ regime seen in the dotted curve and in the insert of the top frame which shows an expanded version of the low $\nu$ part of the QDOS. The bottom frame includes a $s$-wave component $(\beta \neq 0)$ into the gap function before transformation. The dotted line $(\alpha=\beta=0)$ is unchanged and it is for comparison. All other curves have a $\alpha=0.4$. The solid curve is for $\beta=0.4$, dashed is for $\beta=0.6$, and dash-dotted is for $\beta=0.8$. The splitting of the van Hove singularity not seen in the top frame is clearly visible and is traced directly to $\beta \neq 0$.

0.6 , and 0.8 respectively. The dotted curve has $\alpha=\beta=0$ and is a pure $d$-wave case with no band mass anisotropy and is presented for comparison. The other three curves have $\alpha=0.4$ as well as a finite value of $\beta$. It is obvious that for $\alpha=\beta=0$ we recover the well known result that the QDOS acquires a finite value at $\nu=0$ when resonant scattering is included.

Our results for the absorptive part of the optical conductivity $\sigma_{1}(\nu)$ are presented in figure 5 as a function of the reduced photon energy $\nu / \Delta_{0}$. In the top frame the impurity scattering is in Born approximation and in the bottom frame it is in the resonant scattering limit. The gray curve is the $\left(y y^{\prime}\right)$-component and the short dashed curve is the $\left(x x^{\prime}\right)$-component for $\alpha=0.2$, solid and dotted are the same for $\alpha=0.4$ and dashed and dash-dotted are for $\alpha=0.6$. The inserts give an expanded view of the low frequency part of the results.

The most important idea to keep in mind in analyzing our results for the op- 

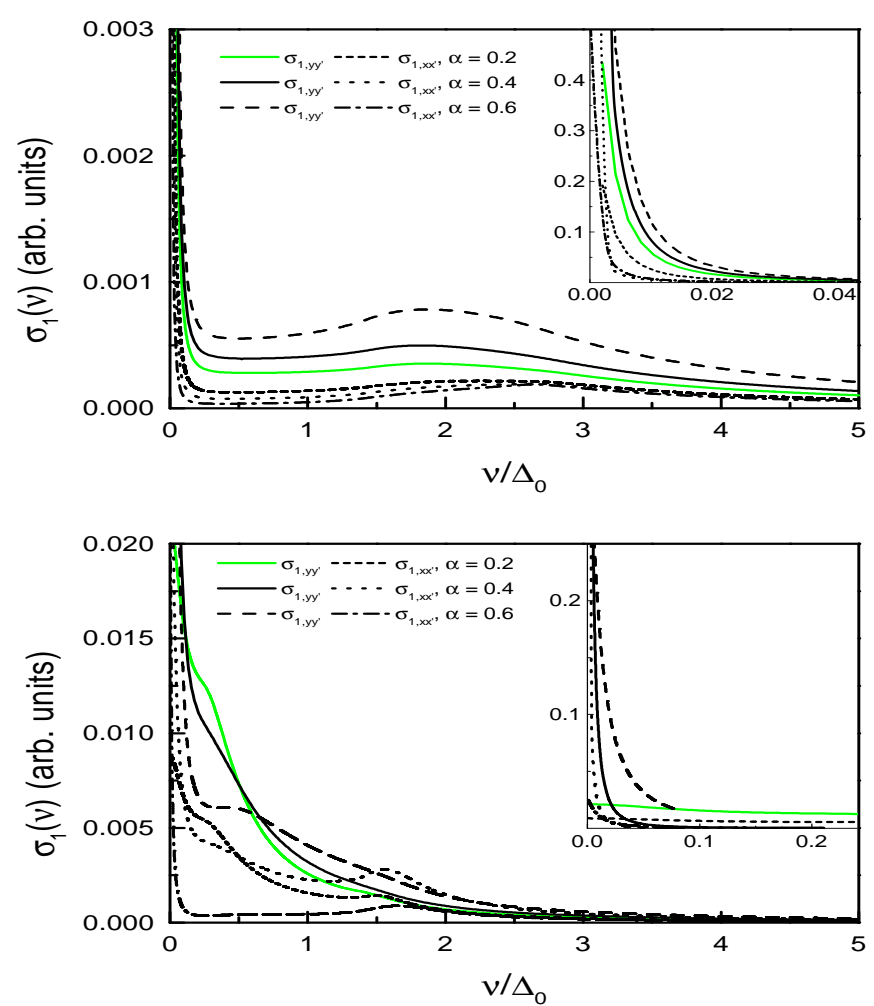

Figure 5. The top frame presents the absorptive part of the infrared conductivity $\sigma_{1}(\nu)$ as a function of the reduced energy $\nu / \Delta_{0}$ for the pure $d$-wave case $(\beta=0)$ with orthorhombic band structure $(\alpha \neq 0)$. The gray line gives $\sigma_{1, x x^{\prime}}$ and the short dashed line gives $\sigma_{1, y y^{\prime}}$ for $\alpha=0.2$, solid and dotted lines correspond to $\sigma_{1, x x^{\prime}}$ and $\sigma_{1, y y^{\prime}}$ for $\alpha=0.4$, and the dashed and dash-dotted ones correspond to $\sigma_{1, x x^{\prime}}$ and $\sigma_{1, y y^{\prime}}$ for $\alpha=0.6$. The insert shows an expanded plot of the low energy part. The scattering is treated in Born approximation. The bottom frame is the same as the top frame but now the scattering is treated in the unitary limit.

tical conductivity is, that in Born scattering, the number of states at the chemical potential goes to zero linearly with decreasing frequency $\nu$ (except at very low temperatures and frequencies not of interest here) while for unitary scattering there is a finite density of states at $\nu=0$ which provides the possibility of transition from the region of $\nu=0$ to all energies in the unoccupied density of states and so these transitions will reflect directly structures in the density of states at the same energies. Such transitions are progressively suppressed, however, as the orthorhombicity $\alpha$ is increased or as the $s$-wave admixture $\beta$ is increased or the scattering strength is reduced towards Born scattering. In such circumstances these transitions can be greatly suppressed and may not lead to significant structures in optical properties at the critical points for the density of states. The structure at twice the gap energy scale reflecting transitions from the main peak in the density of occupied states to the main peak in the unoccupied states can now dominate the optical characteris- 

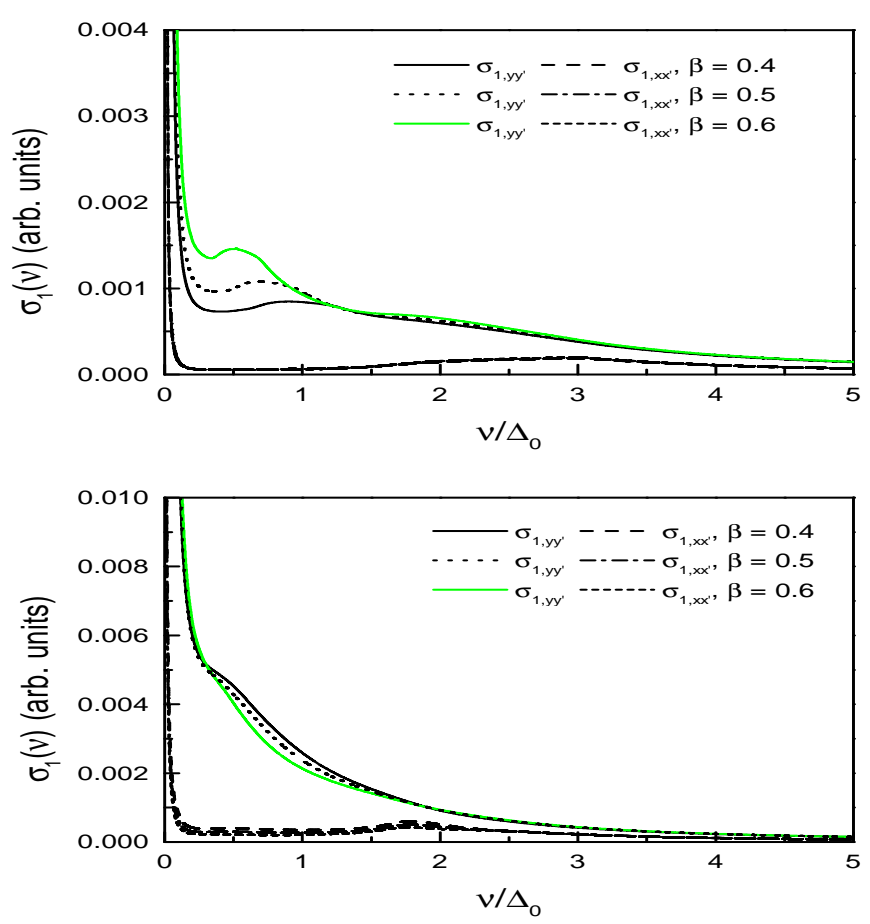

Figure 6. Top frame: the real part of the infrared conductivity $\sigma_{1}(\nu)$ vs. the reduced energy $\nu / \Delta_{0}$ for $\alpha=0.4$ and various values of the $s$-wave admixture $\beta$. The solid, dotted, and gray curves are for $\sigma_{1, y y^{\prime}}$ (along the chains) with $\beta=0.4$, 0.5 , and 0.6 respectively. The dashed, dash-dotted, and short dashed curves are for $\sigma_{1, x x^{\prime}}$. We see in $\sigma_{1, y y^{\prime}}$ the $2 \Delta$ structure at low energies corresponding to the lower energy van Hove peak in the QDOS (see bottom frame of figure 4; this curve applies to resonant scattering, the results for Born scattering are not shown here but are quite similar). In $\sigma_{1, x x^{\prime}}$ we see the high energy van Hove structure of the QDOS at a reduced energy near 3.0. Bottom frame: same as for the top frame but now the impurity scattering is treated in the resonant limit. As before, structures are seen in the $\sigma_{1, x x^{\prime}}$ curves but now around a reduced energy of 1.8.

tics. As far as we are dealing with an orthorhombic system, an extra factor of the $x$ - and $y$-components of the Fermi velocity will enter the conductivity but not the QDOS. This leads to different characteristics in $x$ - and $y$-directions and the structures expected on the basis of the QDOS alone get shifted somewhat by the extra Fermi velocity weighting.

The top and bottom frame of figure 6 present results for the real part of the infrared conductivity with finite band structure orthorhombicity included with $\alpha=$ 0.4 while at the same time some $s$-wave part is included through $\beta$. Three values are considered, namely $\beta=0.4,0.5$, and 0.6. There are 6 curves, solid, dotted, and gray for $\sigma_{1, y y^{\prime}}(\nu)$, dashed, dash-dotted, and short dashed for $\sigma_{1, x x^{\prime}}(\nu)$ in order to increase $\beta$. The top frame is for Born impurity scattering. On comparing solid and dotted curves for $\alpha=0.4$ with the top frame of figure 5 we see that including a 
finite value of $\beta$ has not changed the conductivity in the $\left(x x^{\prime}\right)$-direction by much, although, the peak around $\nu / \Delta_{0}=2.8$ has been shifted a little towards higher values $(\sim 3.0)$. In comparison, the effect of finite $\beta$ has had a drastic effect on the $\sigma_{1, y y^{\prime}}(\nu)$ characteristic which now shows new structures at low energies just above the rapid Drude like rise at $\nu \rightarrow 0$. These structures are traced to the low energy van Hove singularities seen in the bottom frame of figure 4 and are a result of the admixture of a $s$-wave component to the gap function. They are not present when band structure anisotropy alone is accounted for and thus offer the possibility of distinguishing between the two effects and measuring the amount of $s$-wave admixture through optical methods.

If resonant impurity scattering is considered instead of Born scattering we get the results shown in the bottom frame of figure 6. Again, the structure in the curves for $\sigma_{1, x x^{\prime}}(\nu)$ has moved from $\nu / \Delta_{0}=3.0$ or so to 1.7 or so just as in the bottom frame of figure 5 . The curves in the $\left(y y^{\prime}\right)$-direction show little sign of the low energy van Hove structure and this case is not as favourable as the Born scattering case for measuring the value of $\beta$. On the other hand, they show much more spectral weight at low $\nu$.

It is now, of course, quite interesting to compare theoretical predictions of this model with experimental data for the orthorhombic superconductor YBCO. According to Schürrer et al. [11] the anisotropy parameter $\alpha=0.41$ is found from zero-temperature penetration depth measurements by Bonn et al. [15] and the value $\beta=-0.25$ was found to allow the best possible fit to the temperature dependence of the low temperature dependence of the London penetration depth. These parameters are used to calculate the optical conductivity of an orthorhombic $d$-wave superconductor and figure 7 presents a comparison of these results with experimental data reported by Wang et al. [14] for a nominally clean, detwinned YBCO single crystal. On the horizontal scale these data have been scaled to a value of $\Delta_{0}$ which corresponds to a ratio of $2 \Delta_{0} / k_{\mathrm{B}} T_{\mathrm{c}} \approx 10$. The arrow indicates the data point used to fit the experimental data on the vertical scale.

The first thing to be noted is the maximum at $\nu / \Delta_{0}$ slightly above 2 in the dashed curve for $\sigma_{1, x x^{\prime}}$. It is not present in the solid curve for $\sigma_{1, y y^{\prime}}(\nu)$. The energy scale defined in the dashed curve is twice the value of the low energy van Hove structure seen in the QDOS shown in the bottom frame. There is no discernible structure (corresponding to the second, high energy van Hove structure in $N(\nu) / N(0)$ ) seen in the conductivity curve so that this set of parameters is not favourable for measuring the $s$-wave admixture in the gap function. We note several aspects of the top frame of figure 7. First the peak in $\sigma_{1, y y^{\prime}}(\nu)$ has moved to lower frequency $\left(\nu / \Delta_{0} \sim 1.5\right)$ as compared with the $\sigma_{1, x x^{\prime}}$-case $\left(\nu / \Delta_{0} \sim 2\right)$. This softening is observed in the data of Wang et al.[14] on untwinned pure single crystals of YBCO shown. Also the depth of the dip below $\nu=\Delta_{0}$ is more pronounced in our work in the $\left(x x^{\prime}\right)$ than in the $\left(y y^{\prime}\right)$ direction and this is in qualitative agreement with the experiment. While the qualitative agreement with the data is good for the $\left(x x^{\prime}\right)$-component it is less good for the $\left(y y^{\prime}\right)$-component. In particular the large peak in the data around $\nu / \Delta_{0}=0.6$ is not in our theoretical calculations. Nevertheless, we can conclude that 

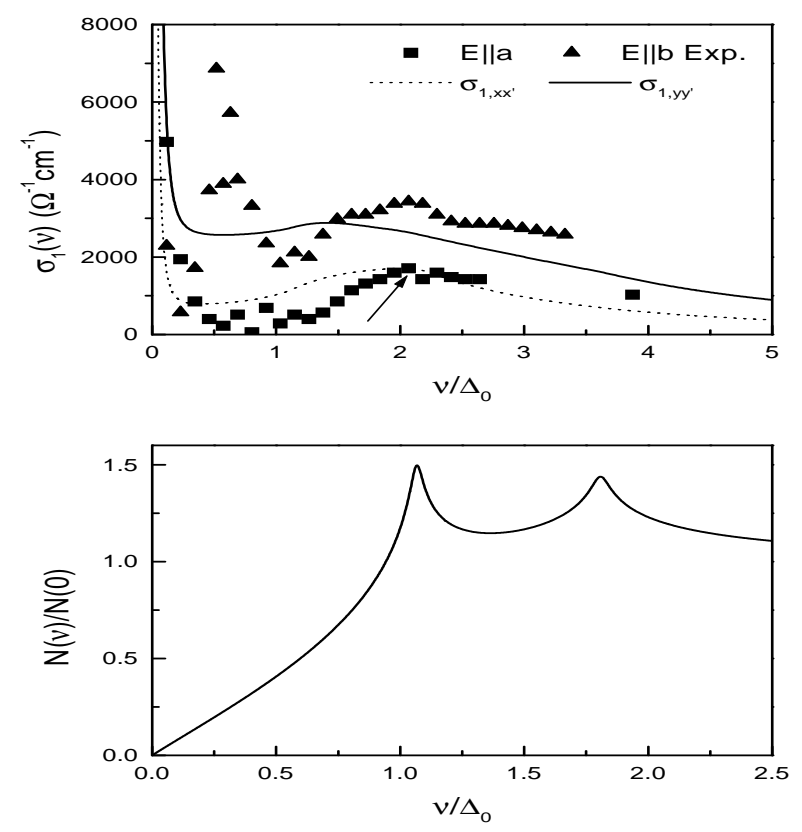

Figure 7. Top frame: the real part of the infrared conductivity $\sigma_{1}(\nu)$ vs. reduced frequency $\nu / \Delta_{0}$ for the case $\alpha=0.41$ and $\beta=-0.25$. The dotted curve is for $\sigma_{1, x x^{\prime}}(\nu)$, the solid one is for $\sigma_{1, y y^{\prime}}$. The structure slightly above $\nu / \Delta_{0}=2$ reflects the low energy van Hove structure in the QDOS shown in the bottom frame. The position of this structure is displaced slightly upwards in the $\sigma_{1, x x^{\prime}}(\nu)$ curve. It is not seen in the $\sigma_{1, y y^{\prime}}(\nu)$ curve which has got a peak at lower frequencies. Also included are experimental results reported by Wang et al.[14] for untwinned YBCO single crystals. The solid squares show $\sigma_{1}(\nu)$ data in $a$-direction and the solid triangles $\sigma_{1}(\nu)$ show data in $b$-direction. The arrow indicates the data point used to fit our theoretical results. Bottom frame: the superconducting state QDOS $N(\nu)$ normalized to its normal state value at the Fermi level $N(0)$ as a function of reduced energy $\nu / \Delta_{0}$.

the orthorhombic $d$-wave model is in a rather good qualitative agreement with the experiment.

\section{Conclusion}

It is one of the main results of this study that the properties of the optical conductivity in superconductors with a mixed symmetry order parameter are explained by the fact that in the $(s+\mathrm{i} d)$-symmetric case there is always a gap in the QDOS for all possible $s$-wave admixtures while the orthorhombic $d$-wave case is characterized by an order parameter with nodes on the Fermi surface for all reasonable values of the mass anisotropy parameter $\alpha$. Additional splitting of the van Hove singularities which is reflected by additional structure in the optical conductivity occurs for further $s$-wave contributions to the order parameter which are characterized by a 
$\beta \neq 0$.

We also considered both the effect of Born and of resonant impurity scattering. The main difference between these two limits is that while for Born impurity scattering the structure in the electromagnetic response due to the corresponding structures in the QDOS are to be found on a $2 \Delta_{0}$ scale, in the resonant case they are to be found on the scale of $\Delta_{0}$ instead. For orthorhombic systems, however, these structures can get shifted in energy or might even not appear because of the extra Fermi velocity weighting factors in the conductivity. Still, structures can be identified which correspond to the van Hove structures. Also, the $x$-axis conductivity is seen to show a more "s-wave"-like behaviour as compared to the $y$-axis which is more " $d$-wave"-like.

\section{Acknowledgements}

Work supported in part by Fonds zur Förderung der wissenschaftlichen Forschung (FWF), Vienna, Austria under contract No. P11890-NAW.

\section{References}

1. Krishana K., Ong N.P., Li Q., Gu G.D., Koshizuka N. Plateaus observed in the field Profile of thermal conductivity in the superconductor $\mathrm{Bi}_{2} \mathrm{Sr}_{2} \mathrm{CaCu}_{2} \mathrm{O}_{8}$. // Science, 1997, vol 277, p. 83-85.

2. Kouznetsov K.A., Sun A.G., Chen B., Katz A.S., Bahcall R., Clarke J., Dynes R.C., Gajewski D.A., Han S.H., Maple M.B., Giapintzakis J., Kim J.-T., Ginsberg D.M. caxis josephson tunnelling between $\mathrm{YBa}_{2} \mathrm{Cu}_{3} \mathrm{O}_{7-\delta}$ and $\mathrm{Pb}$ : Direct Evidence for Mixed Order Parameter Symmetry in a High- $T_{\mathrm{c}}$ Superconductor. // Phys. Rev. Lett., 1997, vol 79, p. 3050-3053.

3. Covington M., Aprili M., Paraoanu E., Greene L.H., Xu F., Zhu J., Mirkin C.A. Observation of surface-induced broken time-reversal symmetry in $\mathrm{YBa}_{2} \mathrm{Cu}_{3} \mathrm{O}_{7}$ tunnel junctions. // Phys. Rev. Lett., 1997, vol 79, p. 277-280.

4. Fogelström M., Rainer D., Sauls J.A. Tunnelling into current-carrying surface states of high- $T_{\mathrm{c}}$ superconductors. // Phys. Rev. Lett., 1997, vol 79, p. 281-284.

5. Timusk T., Tanner D. Optical properties of high temperature superconductors. - In: Physical Properties of High Temperature Superconductors III, ed. Ginsberg D.M., Singapore, World Scientific, 1992, p. 363-469.

6. Palumbo M., Graf N.J. Electromagnetic absorption in an anisotropic layered superconductor. // Phys. Rev B, 1996, vol 53, p. 2261-2264.

7. Basov D.N., Liang R., Bonn D.A., Hardy W.N., Dabrovski B., Quijada M., Tanner D.B., Rice J.P., Ginsberg D.M., Timusk T. In-plane anisotropy of the penetration depth in $\mathrm{YBa}_{2} \mathrm{Cu}_{3} \mathrm{O}_{7-\delta}$ and $\mathrm{YBa}_{2} \mathrm{Cu}_{4} \mathrm{O}_{8}$. // Phys. Rev. Lett., 1995, vol 74, p. 598-601.

8. Preosti G., Palumbo M. Penetration depth measurements as a test for time-reversal breaking states in unconventional superconductors. // Phys. Rev. B, 1997, vol 55, p. 8430-8436.

9. Ambegaokar V. The Green's function method. - In: Superconductivity I, ed. Parks R.D., New York, Marcell Dekker, 1969, p. 259-319. 
10. Schürrer I., Schachinger E., Carbotte J.P. Optical conductivity of superconductors with mixed symmetry order parameters. // Physica C, 1998, in print.

11. Schürrer I., Schachinger E., Carbotte J.P. Optical conductivity in orthorhombic $d$ wave superconductors. // Phys. Rev. B, 1998, submitted.

12. Lee W., Rainer D., Zimmermann W. Holstein effect in the far-infrared conductivity of high- $T_{\mathrm{c}}$ superconductors. // Physica C, 1989, vol 159, p. 535-544.

13. Puchkov A.V., Basov D.N., Timusk T. The pseudogap state in high- $T_{\mathrm{c}}$ superconductors: an infrared study. // J. Phys.: Condens. Matter, 1996, vol 8, p. 10049-10082.

14. Wang N.L., Tajima S., Hauff R., Rykov A.I., Mimura T. Optical study of pair-breaking effect in Zn-substituted $\mathrm{YBa}_{2} \mathrm{Cu}_{3} \mathrm{O}_{7-\delta}$. // J. Phys. Chem. Solids, 1998, in print.

15. Bonn D.A., Kamal S., Zhang K., Liang R., Hardy W.N. The microwave surface impedance of $\mathrm{YBa}_{2} \mathrm{Cu}_{3} \mathrm{O}_{7-\delta}$. // J. Phys. Chem. Solids, 1995, vol 56, p. 1941-1943.

\title{
Оптична провідність у надпровідниках 3 параметром порядку займаної симетрії
}

\author{
І.Шюррер, Е.Шахінґер \\ Інститут теоретичної фізики, Технічний університет м. Грац, \\ Австрія, А-8010 Грац, вул. Петерсгассе, 16 \\ Отримано 21 квітня 1998 р.
}

Розраховані дійсна і уявна складові частини оптичної провідності та інтенсивність оптичного розсіяння у надпровідниках з параметром порядку змішаної $s+d$ та $s+\mathrm{i} d$ симетрії для різної величини змішування і для помірних концентрацій домішок у двох граничних випадках борнівського (слабого) та резонансного (одноразого) розсіяння. Спостерігаються значні відмінності оптичної провідності між граничними випадками для розсіяння на домішках та зі зміною симетрії параметра порядку. Передбачення теорії порівнюються з експериментальними даними.

Ключові слова: високотемпературна надпровідність, змішана симетрія, параметр порядку, оптична провідність

PACS: $74.20 . F g, 74.25 . N f, 74.72 .-h$ 